\title{
Vigilância sanitária de uma cidade metropolitana do sul do Brasil: implantação da gestão plena e efetividade das ações
}

\author{
Health surveillance in a city in the south of Brazil: implantation \\ of the descentralization and effectiveness of the actions
}

Jorcen Simon de Souza ${ }^{1}$ Airton Tetelbom Stein ${ }^{1}$
${ }^{1}$ Programa de PósGraduação em Saúde Coletiva, Universidade Luterana do Brasil. Av. Farroupilha 8001/Prédio 14/227, Bairro São José. 92425-900 Canoas RS. jorcemss@yahoo.com.br
Abstract Thisstudy evaluated the effectiveness of the health surveillance actions in Canoas/RS during the implantation of the local health management system and characterized the profile of the health agents by means of a cross-sectional descriptive study. The target population consisted of all the 12 health agents of theteam. Thedata were collected using a self-applicablequestionnaire(Essential Public Health Function no6) with performance classifications ranging from 0 to 1 ; interview with the direction of the department; and survey of the service reports. The main results obtained through statistical analysis and interpre tation showed that the management model together with the technical commitment of the health agents allowed for an effective implantation of the decentralized, local management; most agents were females; $66.7 \%$ had a mean age of 46 years $( \pm 7.5)$; and $58.31 \%$ of them had higher education and an income of more than five minimum wages; the Essential Public Health Function n-6 measured 0.7 as final average. It is concluded that the health surveillance actions during the decentralization process were effective, showing a performance above the average in relation to the used instrument. The structure of the service and the profile of the health agents are similar to those found in other health surveillance departments. Key words $\mathrm{H}$ ealth surveillance, $\mathrm{H}$ ealth services evaluation, Public health, Decentralization
Resumo Neste estudo, avaliou-se a efetividade das ações do departamento de vigilância sanitária deCanoas, Rio Grande do Sul, na implantação da gestão plena do sistema municipal, caracterizando-seo perfil desses agentespor delineamento transversal descritivo. Todos os fiscais (12) formaram a população-alvo. Osdadosforam coletados por questionário auto-aplicável (Função Essencial deSaúde Pública no 6) com classificação de performance de 0 a 1, entrevista com a direção do departamento e levantamento dos registros do serviço. O btiveramse como principais resultados, por estatística e interpretação, que o modelo de gestão do serviço, associado ao comprometimento técnico dos fiscais, proporcionou efetiva implantação da gestão plena; os fiscais apresentavam maioria feminina (66,7\%) , idademédia de 46 anos $( \pm 7,5)$ e 58,31\% possuíam nível superior e renda maior que cinco saláriosmínimos; a Função Essencial deSaúdePública no 6 mediu 0,7 como média final. Conclui-se que 0 departamento apresentou efetividade nas ações de vigilância sanitária durante a descentralização, apresentando desempenho acima da mé dia frente ao instrumento utilizado, além do serviço constar de estrutura e grupo de fiscais com perfil semelhante a outras equipes de vigilância sanitária.

Palavras-chave Vigilância sanitária, Avaliação de serviços de saúde, Saúde coletiva, D escentralização 
Introdução

Em 1988, a Constituição Federal ${ }^{1}$ afirmou ser a saúde um direito de todos e destacou as atribuições da vigilância sanitária como obrigação do Estado, produzindo intensa atividade regulatória $^{2}$. D esde então, as ações de vigilância sanitária são consideradas as atividades preventivas mais eficientes para a concretização do direito à saúde da população no campo de atuação do Sistema Ú nico de Saúde (SUS) ${ }^{3}$, a ponto de ser possível afirmar que as competências do SUS, como um todo, apontam praticamente todas as atividades do encargo da vigilância sanitária ${ }^{4}$. N este âmbito, esse setor representa um influente mecanismo para articular níveis de poderes governamentais e para impulsionar ações e movimentos de participação social principalmente em razão do papel que exerce na regulamentação e fiscalização das relações entre produção e comercialização ${ }^{5}$. Com efeito, a abrangente e complexa atividade de vigilância sanitária é conceituada atualmente na Lei Orgânica da Saúde - Lei no 8.080, de 19 de Setembro de $1990^{6}$, como um conjunto de ações capazes de eliminar, diminuir ou prevenir riscos à saúde e de intervir nos problemas sanitários decorrentes do meio ambiente, da produção e circulação de bens e da prestação de serviços de interesse da saúde, abrangendo o controle de bens de consumo que, direta ou indiretamente, se relacionam com a saúde da população.

Observa-se, então, que o campo de atuação da vigilância sanitária é complexo em razão do constante modo de produção capitalista de mercadorias e serviços, respondendo à lógica do sistema produtivo. E, por outro lado, delicado, do ponto de vista dos interesses envolvidos, pois a defesa e a proteção da saúde coletiva na maioria das vezes significam clara oposição a interesses hegemônicos que detêm grande parcela de poder na sociedade 7 . D esta forma, a plena estruturação da vigilância sanitária torna-se requisito fundamental para a efetiva implantação do SUS, principalmente em virtude de seu poder normativo e fiscalizatório aplicado aos serviços e na qualidade dos insumos terapêuticos que estes consomem ${ }^{8}$.

Todavia, a qualidade da gestão dos serviços do SUS pode ser amplamente aperfeiçoada por meio do uso adequado dos instrumentos de que a vigilância sanitária dispõe $e^{9}$. Além disso, a qualidade das relações entre os prestadores desses serviços e a população que os recebeéimpulsionada pela ação da fiscalização sanitária. Estas ações têm o propósito de implementar concepções e atitudes éticas a respeito da qualidade das relações, dos processos produtivos, do ambientee dos serviços de saúde ${ }^{10}$. Entendendo-sequea efetiva gestão das ações de vigilância sanitária apresenta potencial importantíssimo tanto para a reversão do antigo modelo assistencial de saúde quanto para alimentar ou enriquecer os processos indispensáveis à construção e garantia da qual idade de vida dos cidadãos ${ }^{11}$ e ser, de fato, assumida como uma atividade importantedo planejamento eprogramação de saúde no município ${ }^{12}$.

Descentralização municipal da saúde e as ações de vigilância sanitária

No limiar do processo de descentral ização das ações em saúde, com ênfase para os municípios, a definição de competências entre as três esferas do poder alcança também os serviços de vigilância sanitária ${ }^{13}$, por onde a gestão municipal deve fazer executar as atribuições previstas nas N ormas O peracionais Básicas (NOB), editadas pelo poder executivo, através de repasses financeiros instituídos pelo Piso Básico de Vigilância Sanitária (PBVS). As N O B introduzem condições estabelecidas segundo responsabilidades, prerrogativas e mecanismos de financiamento, que o gestor se dispõe a assumir, segundo sua vontade política esuas condições técnicas emateriais, com a aprovação do respectivo Conselho de Saúde ${ }^{14}$.

Atualmente, a N OB SU S 01/96 15 regula a descentral ização das ações devigilância sanitária, prevendo duas condições de gestão para os municípios: plena da atenção básica e plena do sistema municipal. Todavia, municípios em Gestão Plena do Sistema Municipal (GPSM) detêm responsabilidade pela execução das ações básicas, de mé dia e alta complexidade em vigilância sanitária.

N esta lógica, a implantação da GPSM , como uma etapa do processo de descentralização das ações de saúde, representa a concretização da municipalização da saúdee constitui subsídio importantepara o planejamento, gerenciamento equalidade dos serviços de assistência médica, e para o controle de qualidade de produtos e serviços de saúde, todos inerentes à vida da população $0^{16}$.

Atualmente, as alternativas de descentralização das ações de vigilância sanitária para os municípios passam por novas propostas de modelos de gestão aplicáveis ao setor eque pretendem propor, em última análise, menos desperdício e melhoria da qualidade dos serviços oferecidos ${ }^{17}$. 
Avaliação de serviços de saúde

Outrossim, a avaliação em saúde produz informações quanto à adequação, efeitos e custos associados ao uso de tecnologias e tipos de gestão ${ }^{18}$. D onabedian ${ }^{19}$ propõeum conceito degrandeimportância, o de padrão ou categoria, salientando-se que a descentralização e 0 acesso são categorias universalmente utilizadas em análises de qualidade de serviços de saúde, aparecendo como promissoras na construção de um mode lo ideal deavaliação, considerando queum serviço descentralizado possibilita o equilíbrio entre possibilidade, obrigações e poderes do nível central elocal20.

As avaliações de serviços de saúde mais conhecidas, utilizando o modelo de Donabedian ${ }^{21}$, possuem ênfase na tríade "eficácia, efetividade e eficiência", objetivando a otimização dos procedimentos eserviços. A medida de efetividadetem, no seu termo, significado aproximado ao de eficácia, mas com a diferença de quenão se refere às situações "ideais" de avaliação, mas sim a condições "normais" de uso do serviço a ser avaliado, assim como verificado na fiscalização sanitária. No que tange a esta realidade, a efetividade diz respeito à utilidade ou ao benefício, de um produto ou serviço de saúde, quando usado pela população ${ }^{22}$. O utrora, Jam ${ }^{23}$ propõe que a efetividade de um serviço de saúde é o grau no qual os desfechos desejados são atingidos. Assim, temse a efetividade como medida indicada por referencial teórico para avaliar serviços de saúde, incluindo o de vigilância sanitária, contemplando suas especiais características de execução e ação.

N este contexto, o objetivo do presente estudo éavaliar a efetividade das ações desenvolvidas no serviço de vigilância sanitária do município deCanoas, Rio Grande do Sul, duranteo processo de implantação da GPSM , pela ótica dos seus próprios profissionais fiscais, bem como conhecer o perfil e características destes agentes.

\section{M etodologia}

0 objeto desteestudo foi o departamento de vigilância sanitária do município de Canoas, Rio Grande do Sul, órgão departamental da Secretaria Municipal de Saúde. Canoas é o município mais populoso da Região Metropolitana, com

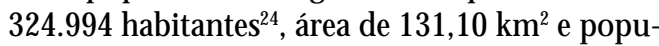
lação $100 \%$ urbana ${ }^{25}$. 0 serviço de vigilância sanitária de Canoas foi instituído como departamento em junho de 2000 pela Lei M unicipal $n-4.458$.
A criação do departamento foi motivada em virtude da readequação das funções de vigilância sanitária, até então executadas pelo Estado. Dentro do organograma de gestão municipal, o departamento de vigilância sanitária évinculado diretamente à Secretaria Municipal da Saúde, em conjunto com outros quatro departamentos. A população-alvo do estudo foi composta pelo diretor do departamento e servidores do setor de fiscalização, que formavam uma equipe de doze profissionais, em todo o seu grupo. No período da pesquisa, durante implantação da GPSM , a fiscalização era realizada totalmente por demanda interna do departamento, que constava de servidores municipais e estaduais ali lotados.

Os aspectos éticos para a pesquisa foram considerados através da aplicação de termo de consentimento livre esclarecido em toda populaçãoalvo, bem como submissão e aprovação pelo Comitê de Ética em Pesquisa da Universidade Luterana do Brasil.

Os dados foram coletados entre novembro e dezembro de 2005, em visitas ao ambiente detrabalho dos sujeitos do estudo. A coleta de dados ocorreu em duas fases, sendo a primeira com coleta de dados primários através da aplicação de instrumento de pesquisa auto-aplicável distribuído aosfiscais sanitários, ea partir de entrevista com a direção do departamento para obtenção das informações a respeito do processo de descentralização. A segunda fase, com coleta de dados de fontes secundárias, ocorreu por levantamento documental de registros e informações concedidas pelo próprio serviço, referente aos procedimentos realizados pelo departamento durante a implantação da GPSM .

Para a avaliação da efetividade do serviço, foi realizado um estudo com delineamento transversal, utilizando abordagem quantitativa descritiva. 0 instrumento de pesquisa auto-aplicável foi utilizado em toda a população-alvo do estudo e composto de dois segmentos, um segmento voltado a coletar dados para a caracterização dos recursos humanos, que incluía as seguintes variáveis: idade, sexo, estado civil, escolaridade, renda, satisfação quanto à renda, função exercida, regime de trabalho, tempo que trabaIha na fiscalização, satisfação quanto à formação esatisfação quanto a disponibilização de treinamentos na área que atua. 0 outro segmento foi formado pelo instrumento denominado Função Essencial de Saúde Pública - FESP - № 6, formulado pela Organização Pan-Americana de Saúde (OPAS), e que visa medir o desempenho do fortalecimento dacapacidadeinstitucional de 
regulamentação e fiscalização em saúde pública. 0 questionário FESP foi validado pela OPAS durante os meses de abril e maio de 1999 em três países: Bolívia, Colômbia e Jamaica ${ }^{26}$.

A FESP $n-6$ avalia o fortalecimento da capacidade institucional do gestor de regulamentar e fiscalizar a saúde pública no local de atuação. Seu foco principal baseia-seem avaliar a execução de todas as atividades de regulamentação do serviço defiscalização da vigilância sanitária de forma oportuna, correta, consistente e completa ${ }^{27}$. Possui quatro indicadores, que avaliam a infra-estrutura, as capacidades, os processos e os resultados envolvidos no desempenho da função. Cada um deles apresenta um padrão que descreve o "ótimo aceitável" de cumprimento para 0 indicador respectivo eum conjunto de medições e submedições que admitem respostas afirmativas ou negativas, sendo inaceitável uma resposta parcial ${ }^{28}$. Para 0 desenvolvimento da pesquisa, foram utilizados trêsindicadores dos quatro existentes no instrumento para melhor responder à questão de pesquisa (Quadro 1 ). Os resultados obtidos pela medição do instrumento FESP $n \circ 6$ foram construídos a partir do cálculo das médias resultantes das 53 variáveis totais que cada questionário possui. A média foi calculada, correspondendo à soma dos valores respectivos às respostas "sim", divididos pelo número total de questões que cada indicador compunha. 0 Quadro 1 identifica os indicadores FESP utilizadose descreve o objetivo direto da medição de cada indicador, demonstrando o número total de questões que os compõem.

A FESP $\mathrm{n}-6$ apresenta como característica metodológica 0 consenso da população aplicada, em quea "opinião especializada" de quem responde ao instrumento evidencia no final de sua aplicação um valor de medida que identifica o desempenho obtido. Para esta interpretação, considerou-se $0,50(50 \%)$ como ponto médio e classificação de performances conforme escaladeresultados, em quea pontuação de 0,0 a 0,25 representa desempenho mínimo; 0,26 a 0,50, desempenho médio inferior; 0,51 a 0,75 , desempenho médio superior e 0,76 a 1,0, desempenho ótimo ${ }^{29}$.

Os dados foram analisados através de programas estatísticos na seguinte seqüência: Epidata $3.1{ }^{30}$, Epidata Analysis $\mathbb{B}^{11}$ e SPSS $\AA^{32}$, obtendo-se assim a estatística descritiva. A apresentação dos dados deu-se de forma tabular através de freqüências e medidas de tendência central. Foram realizadas análises bivariadas entre as variáveis que visavam à caracterização dos recursos humanos, considerando-se estatisticamente significativos onde $p>0,05$. U tilizou-se cálculo de médias e desvio padrão para as variáveis contínuas e proporção para as variáveis categóricas. Procedeu-seà organização do conjunto de material de pesquisa obtido através das entrevistas e da análise documental, buscandose confrontar os resultados encontrados com 0 referencial teórico afeto à área de vigilância sanitária e a adesão ao processo de descentralização.

Quadro 1. Indicadores do instrumento FESP $n \cong 0$, seus objetivos de medição e respectivo número de variáveis.

\begin{tabular}{|l|l|c|}
\hline \multicolumn{1}{|c|}{$\begin{array}{c}\text { Indicadores FESP } \\
\text { no } 6\end{array}$} & \multicolumn{1}{|c|}{ Objetivo da medição } & $\begin{array}{c}\text { Número de } \\
\text { variáveis }\end{array}$ \\
\hline FESP 6.2 & $\begin{array}{l}\text { Avaliar o serviço de vigilância sanitária quanto ao } \\
\text { cumprimento das normas em saúde }\end{array}$ & 18 \\
\hline FESP 6.3 & $\begin{array}{l}\text { Analisar os conhecimentos, habilidades e mecanismos do } \\
\text { serviço de vigilância sanitária para revisar e fazer cumprir o } \\
\text { marco regulatório }\end{array}$ & 23 \\
\hline FESP 6.4 & $\begin{array}{l}\text { Avaliar a disposição de assessoria e apoio técnico do nível } \\
\text { subnacional direto ao serviço de vigilância sanitária para } \\
\text { fiscalização de leis e regulamentações }\end{array}$ & 12 \\
\hline & \begin{tabular}{l} 
TOTAL \\
\hline
\end{tabular} \\
\hline
\end{tabular}

Fonte: $\mathrm{OPAS}^{27}$. 


\section{Resultados}

Os dados obtidos como resultados estão relacionados à descrição do serviço de vigilância sanitária durante o processo de implantação da GPSM e sua estrutura administrativa organizacional; quanto à descrição da população-alvo referente à caracterização do perfil dos trabalhadores do serviço; e considerando as médias finais obtidas através da medição do instrumento FESP $n^{\circ} 6$.

\section{Descrição \\ do serviço de vigilância sanitária}

0 departamento foi incorporado à GPSM através da Portaria $n$ ㅇ 1.513/GM, de 02 de setembro de 2005, passando a exercer todas as atribuições e competências de baixa e média complexidade, e algumas de alta complexidade. Em setembro de 2006, conforme pactuação regi da pela Comissão Intergestores Bipartite (CIB), passou a realizar as ações de alta complexidade com a inclusão da atividade de aprovação de projetos arquitetônicos de indústrias e de estabelecimentos hospitalares, sem o intermédio total da respectiva Coordenadoria Regional de Saúde. A Tabela 1 apresenta algumas das principais atividades desenvolvidas pelo departamento, durante o processo de implantação da GPSM .

Observou-se que o serviço apresenta gestão própria eindependente, com poder de decisão em suas ações, desenvolvendo internamente seu orçamento e plano de aplicação. No período de pesquisa, o departamento era composto por servidores de nível fundamental, médio e superior, perfazendo um total de cento e cinqüenta (150) profissionais das mais diversas áreas de formação, sendo a equipe técnica de fiscalização composta por doze (12) fiscais de nível médio esupe rior. Deste grupo, aponta-se um (01) profissional como chefe de fiscalização e outro (01) sendo o diretor do departamento, ambos constando como equipe técnica e de gestão. Para a pesquisa, os fiscais foram classificados em dois grandes grupos: fiscais de alimentos e fiscais de estabelecimentos de saúde. Destaca-se que todos os profissionais classificados como fiscais deestabelecimentos de saúdetambém realizavam inspeções relacionadas a alimentos à medida que se faziam necessárias. Entretanto, os fiscais de alimentos não executavam ações em estabelecimentos de saúde.

Todos profissionais realizavam fiscalizações programadas conforme planejamento anual baseado em Termo de Ajuste de Conduta (TAC), bem como as derivadas das necessidades de atendimento aos processos encaminhados. Tinham como conduta padrão e norma interna do departamento realizar as inspeções somente em duplas, dispondo de postura baseada na educação e exigência das competências legais específicas em cada tipo de vistoria.

No que tange ao processo de descentralização das ações de vigilância sanitária, passando da gestão de aten ção básica para a GPSM , segundo a direção do departamento, as principais dificuldades encontradas foram: (1) montagem do processo administrativo para solicitação da descentralização para a gestão plena; (2) contratação de pessoal especializado, frente ao pouco número de profissionais com formação técnica em vigilância sanitária; (3) utilização dos recursos disponíveis para contratação destes profissionais em decorrência do período eleitoral que abrangeu este momento; (4) realização de concurso público para efetivação de pessoal para trabalhar no respectivo departamento.

Tabela 1. Procedimentos realizados pelo departamento de vigilância sanitária de Canoas, Rio Grande do Sul, no período anual de 2005.

\begin{tabular}{|c|c|}
\hline Procedimentos realizados & Quantidade \\
\hline Estabelecimentos vistoriados & 3.517 \\
\hline $\begin{array}{l}\text { Notificações contra } \\
\text { estabelecimentos }\end{array}$ & 520 \\
\hline Alvarás de saúde requeridos & 508 \\
\hline Alvarás de saúde expedidos & 459 \\
\hline $\begin{array}{l}\text { Termos de apreensão } \\
\text { aplicados }\end{array}$ & 12 \\
\hline Produtos apreendidos & $\begin{array}{l}474 \text { unidades de ovos } \\
210 \text { unidades de pães crus } \\
109 \text { unidades de pães cozidos } \\
142 \mathrm{~kg} \text { de carne bovina } \\
3.100 \text { g de confeito } \\
15 \mathrm{~kg} \text { de maionese caseira }\end{array}$ \\
\hline Autos de infração aplicados & 387 \\
\hline Interdições & 11 \\
\hline $\begin{array}{l}\text { Denúncias recebidas/ } \\
\text { Denúncias atendidas }\end{array}$ & 240 \\
\hline
\end{tabular}

Fonte: Departamento de vigilância sanitária de Canoas, Rio Grande do Sul, 2005. 
Como facilitadores no processo de descentralização, cita-se o interesse do gestor de saúde do município e do departamento para o desenvolvimento deste processo; a disponibilidade de uma equipe já capacitada para realizar as atividades deste tipo de gestão; e 0 apoio do ConseIho Municipal de Saúde.

Caracterização do perfil da população-alvo

Dos doze profissionais entrevistados, $41,69 \%$ foram classificados como fiscais de alimentos e $58,31 \%$, como fiscais de estabelecimentos de saúde, sendo que, destes, $16,6 \%$ desenvolviam suas funções como chefe de fiscalização (8,33\%) e diretor de departamento $(8,33)$. Os profissionais com nível superior representavam $58,31 \%$ da população estudada (Tabela 2).

Destes profissionais, 91,7\% relataram ser estatutários e todos realizavam um regime de trabalho de seis horas diárias, em função do horário defuncionamento do departamento durante a pesquisa. 0 tempo médio de trabalho destes profissionais como fiscais foi de 8,67 anos $( \pm 8,6)$. A média de idade foi de 46 anos $( \pm 7,5)$, sendo predominante 0 sexo feminino, apresentando percentual de $66,7 \%$ da amostra. Quanto ao estado civil, 58,3\% dos fiscais entrevistados seclassificaram como solteiros, separados ou viúvos.

No que se refere à escolaridade, $66,6 \%$ possuíam formação no ensino superior entre graduação e pós-graduação. Referente à renda,
$58,3 \%$ obtinham renda mensal de mais de cinco salários mínimos, sendo que $66,7 \%$ consideravam que tinham renda adequada em vista das atividades que desenvolviam. Ao utilizar análise bivariada para avaliar a satisfação dos profissionais quanto a sua renda frente às atividades que desenvolviam, verificou-seque $57,1 \%(p=0,36)$ dos profissionais que recebiam maior renda (mais que cinco salários mínimos) estavam satisfeitos. Entretanto, todos os indivíduos que obtinham renda menor que cinco salários mínimos apresentavam insatisfação.

Quanto à escolaridade e renda, observou-se que todos os profissionais que possuíam ensino superior incompleto ou completo, bem como pós-graduação completa, apresentavam renda de no mínimo quatro, chegando a mais de cinco salários mínimos. Contudo, os profissionais com escolaridade atéo ensino médio completo tinham renda máxima de quatro salários mínimos. Verificou-se que os profissionais com maior escolaridade são os que apresentavam maior renda.

Quando questionados se possuíam formação adequada para realizar a função que desenvolviam, $75 \%$ dos entrevistados responderam afirmativamente. Em análise bivariada a esta questão relacionada à escolaridade, todos os profissionais que apresentavam graduação (incompleta ou completa) e pós-graduação completa consideravam que possuíam formação adequada para as atividades que desenvolviam. $E$, dentre os profissionais que possuíam escolaridade

Tabela 2. Quadro profissional dos fiscais do departamento de vigilância sanitária de Canoas, Rio Grande do Sul, durante o período da pesquisa.

Profissionais fiscais do Departamento de Vigilância Sanitária de Canoas

\begin{tabular}{llcr}
\hline Escolaridade de nível superior & Formação profissional & Quantidade & $\%$ \\
Fiscais de estabelecimentos de saúde & Médico & 1 & 8,33 \\
& Enfermeiro & 1 & 8,33 \\
& Fisioterapeuta & 1 & 8,33 \\
& Dentista & 1 & 8,33 \\
& Engenheiro sanitário & 1 & 8,33 \\
Escolaridade de nível médio & Assistente social & 1 & 8,33 \\
Fiscais de alimentos & Pedagogo & 1 & 8,33 \\
& Total & 7 & 58,31 \\
& & & 41,69 \\
& Fiscal sanitário & 5 & 41,69 \\
& Total & 5 & 100
\end{tabular}

Fonte: Departamento de vigilância sanitária de Canoas, Rio Grandedo Sul, 2005. 
até o nível médio, apenas um $(8,3 \%)$ do total de quatro profissionais ( $33,3 \%$ da amostra) que apresentava ensino médio incompleto considerou que possuía formação adequada para o ple no exercício de suas funções $(p=0,01)$.

Com relação a capacitações e treinamentos para a função exercida, também em análise bivariada, apenas $50 \%$ da amostra consideraram que realizavam capacitação suficiente para o pleno desenvolvimento da função, embora $66,7 \%$ compreendessem que existe disponibilização de treinamento nas respectivas áreas de trabalho ( $p=$ $0,01)$.

M edição do desempenho da Função Essencial de Saúde Pública (FESP) $\mathrm{n} \cong 6$

As médias obtidas referentes aos principais indicadores, quando calculadas separadamente, foram de $0,7( \pm 0,21)$ para 0 indicador FESP 6.2 e $0,63( \pm 0,27)$ para o indicador FESP 6.3 , ambos se enquadrando na faixa percentual de desempenho acima da média, e $0,76( \pm 0,28)$ para 0 indicador FESP 6.4, apresentando resultado de ótimo desempenho. A Tabela 3 apresenta as médias obtidas por cada questionário, correspondentes a cada indicador e suas respectivas médias finais, considerando todas as variáveis (53) do instrumento FESP $n$ - 6 .

A média final obtida pela medição do instrumento, quando considerados todos os indicadores no cálculo, foi de $0,7( \pm 0,26)$, classificando-se num resultado de desempenho acima da média (Tabela 3).

Dentrecadaum dostrês indicadoresutilizados para compor o instrumento de avaliação, algumas de suas variáveis apresentam maior relevância do ponto de vista dos objetivos do estudo e da medição de cada indicador, as quais foram selecionadas devido ao desempenho obtido na edição do instrumento. A Tabela 4 apresenta estas variáveis com descrição das freqüências de respostas afirmativas para cada uma, respectivamente.

Pode-se observar que, referente ao indicador FESP 6.2, a variável que apresenta maior percentual, com $83,3 \%$ de respostas positivas, é a que faz referência à agilidade da gestão do serviço para corrigir o abuso de poder dos fiscais. Referente ao indicador FESP 6.3, verifica-se o mesmo percentual de aceitação para a variável que questiona se o departamento dispõe de conhecimentos e recursos para exercer a função fiscalizadora. Quanto ao indicador FESP 6.4, observa-se

Tabela 3. M édias de medição de desempenho do instrumento FESP $n$ ㅇ 6 compilando todos os doze fiscais entrevistados.

Profissionais fiscais do Departamento de Vigilância Sanitária de Canoas

\begin{tabular}{lccc}
\hline Fiscais entrevistados & $\begin{array}{c}\text { Médias indicador } \\
\text { FESP } 6.2\end{array}$ & $\begin{array}{c}\text { M édias indicador } \\
\text { FESP } 6.3\end{array}$ & $\begin{array}{r}\text { M édias indicador } \\
\text { FESP } 6.4\end{array}$ \\
\hline 1 & 0,88 & 0,86 & 0,66 \\
2 & 0,72 & 0,95 & 0,75 \\
3 & 1,00 & 0,86 & 1,00 \\
4 & 0,72 & 0,60 & 1,00 \\
5 & 0,94 & 0,86 & 1,00 \\
6 & 0,61 & 0,08 & 0,75 \\
7 & 0,77 & 0,47 & 1,00 \\
8 & 0,94 & 1,00 & 1,00 \\
9 & 0,38 & 0,43 & 0,58 \\
10 & 0,61 & 0,60 & 0,66 \\
11 & 0,33 & 0,34 & 0 \\
12 & 0,61 & 0,56 & 0,75 \\
Total de variáveis por indicador & 18 & 23 & 12 \\
Médias totais dos indicadores & 0,7 & 0,63 & 0,76 \\
Desvio padrão & 0,21 & 0,27 & 0,28 \\
Média final & 0,7 & & \\
Desvio padrão & 0,26 & & \\
\hline
\end{tabular}


Tabela 4. Principais variáveis de cada indicador do instrumento FESP no 6 e respectivas freqüências de respostas afirmativas.

Principais variáveis dos indicadores FESP $\mathrm{n} \cong 6$

Indicador FESP 6.2 - Avaliar o cumprimento das normas em saúde

\begin{tabular}{llrr}
\hline No variável & & $\mathrm{n} *$ & $\% * *$ \\
\hline 6.2 .1 .4 & A gestão do serviço é ágil para corrigir o abuso de poder dos ficais & 10 & 83,3 \\
6.2 .1 .6 & $\begin{array}{l}\text { A gestão do serviço monitora a oportunidade e a eficiência da } \\
\text { fiscalização }\end{array}$ & 86,7 \\
6.2 .2 & $\begin{array}{l}\text { A gestão do serviço instrui sobre as normas de saúde pública e } \\
\text { estimula seu cumprimento }\end{array}$ & 9 & 75 \\
6.2 .3 & $\begin{array}{l}\text { A gestão do serviço desenvolve e usa política e planos para } \\
\text { prevenir a corrupção no sistema }\end{array}$ & 8 & 66,7 \\
6.2 .3 .3 & $\begin{array}{l}\text { A gestão do serviço contempla medidas para evitar manipulações } \\
\text { de grupos de pressão externos }\end{array}$ & 6 & 50
\end{tabular}

Indicador FESP 6.3 - Analisar conhecimentos, habilidades e mecanismos para cumprir o marco regulatório

\begin{tabular}{|c|c|c|c|}
\hline 6.3.1.2 & $\begin{array}{l}\text { Departamento dispõe de conhecimentos, habilidades e recursos } \\
\text { para exercer a função fiscalizadora }\end{array}$ & 10 & 83,3 \\
\hline 6.3.1.2.1 & $\begin{array}{l}\text { Departamento dispõe de recursos humanos suficientes para } \\
\text { fiscalização }\end{array}$ & 2 & 16,7 \\
\hline 6.3 .1 .2 .3 & Departamento dispõe de recursos financeiros suficientes & 9 & 75 \\
\hline 6.3 .2 & $\begin{array}{l}\text { Departamento dispõe de mecanismos e recursos para fazer } \\
\text { cumprir as normas de saúde }\end{array}$ & 9 & 75 \\
\hline 6.3.3.2 & Cursos e treinamentos são disponíveis atualmente & 6 & 50 \\
\hline
\end{tabular}

Indicador FESP 6.4 - Avaliar a assessoria do Estado no processo de descentralização

$\begin{array}{llrr}6.4 .1 .1 & \begin{array}{l}\text { Autoridade Sanitária Estadual apresenta protocolos para o } \\ \text { desenvolvimento descentralizado de normas }\end{array} & 10 & 83,3 \\ 6.4 .1 .3 & \begin{array}{l}\text { Autoridade Sanitária Estadual apóia com treinamentos em } \\ \text { processos de regulamentação descentralizada }\end{array} & 10 & 83,3 \\ 6.4 .2 & \begin{array}{l}\text { Autoridade Sanitária Estadual orienta e apóia na fiscalização no } \\ \text { âmbito de sua competência }\end{array} & 11 & 91,7 \\ 6.4 .2 .3 & \begin{array}{l}\text { Autoridade Sanitária Estadual apóia com treinamentos em } \\ \text { procedimentos de fiscalização. } \\ \text { Autoridade Sanitária Estadual presta assistência com pessoal } \\ \text { especializado. }\end{array} & 11 & 91,7 \\ & & 10 & 83.3\end{array}$

* número de questionários que continham respostas afirmativas, deum total (n) dedozequestionários; ** percentuais de respostas à opção afirmativa de cada variável.

duas variáveis com percentuais de aceitação de $91,7 \%$, a primeira verifica sea AutoridadeSanitária Estadual orienta e apóia na fiscalização, e a segunda indica se a AutoridadeSanitária Estadual apóia com treinamentos em procedimentos de fiscalização.

\section{Discussão}

Gestão do serviço e implantação da GPSM durante o processo de descentralização

Pode-se observar no presente estudo, frente aos resultados obtidos, que o modelo de gestão do departamento de vigilância sanitária de Canoas, Rio Grande do Sul, com livreinteração com 
- Estado e sintonizado com as premissas da descentralização, somado ao comprometimento técnico de toda a equipe de trabalho em suas ações, proporcionou sustentação para um efetivo processo de implantação da GPSM das ações de vigilância sanitária. Evidentementequea gestão dessas ações, tendo a descentralização como eixo orientador, depende essencialmente, dentre outros aspectos, da clareza e definição de papéis e atribuições dos principais atores sociais envolvidos neste processo, os gestores, bem como o reconhecimento de que é necessária a incorporação de uma atitude de co-responsabilidade na construção de um sistema de saúde integral que visa, acima de tudo, à garantia do direito à saúde dos usuários.

Quanto a administração dos recursos financeiros destinados às ações de vigilância sanitária, verificou-se no departamento-alvo desta pesquisa queal gumas ações ligadas à plena implantação da GPSM já estavam sendo realizadas anteriormenteao efetivo repasse financeiro originado pelo processo de descentralização, visto o poder de decisão do departamento a respeito de seu orçamento e plano de aplicação. Contudo, Juliano et al. ${ }^{33}$ afirmam que, na prática, a descentralização das ações de vigilância sanitária, ainda que incipiente e de forma desarticulada com a instância regional, começa a delinear somente a partir do ple no recebimento dos recursos financeiros veiculados a este processo. Cohen et al..$^{34}$ ainda apontam que o desconhecimento da maioria dos coorde nadores dos departamentos de vigilância sanitária sobre o tipo de gestão municipal e o montante do PBV S resulta em dificuldades no exercício das atribuições recebidas pela descentralização.

E ambos os autores em estudo de seus respectivos Estados, Juliano et al. ${ }^{33}$ na Bahia, e Cohen et al. ${ }^{34}$ no Rio de Janeiro, apontam que, na prática, encontram-se serviços de vigilância sanitária municipais em GPSM sem condições mínimas para o desempenho de suas ações, apesar de todos os repasses advindos das esferas supe riores do poder público, situação esta decorrente de vários motivos, sejam eles resultantes da má aplicação do recurso financeiro, de ineficiência na gestão do serviço ou ausência de um projeto político para uma efetiva gestão descentralizada das ações de vigilância sanitária no município.

Referente à discussão do modo de gestão do departamento objeto deste estudo, porém sob a ótica das ações intersetoriais, ressalta-se a conjugação de esforços deste com outros atores sociais degranderelevância para a efetividade desuas ações, considerando a complexidade que envol- ve o campo da vigilância sanitária, as suas interconexões eo caráter transdisciplinar dos seus objetos de controle. Assim, destaca-se a parceria do departamento de vigilância sanitária em ações conjuntas realizadas com a Brigada Militar e 0 Conselho Municipal de Saúde. Ressaltando-se a reflexão teórica de Augusto ${ }^{12}$ quando afirma que a interdisciplinaridadenos aponta claramenteum caminho no qual poderemos encontrar as possibilidades criativas de solução para os problemas de saúde das populações.

Ainda sobre os aspectos do modo de gestão e atuação do departamento, verificou-se parecer ocorrer articulada integração com a mídia referenteà divulgação de suas ações, eisso, segundo a direção do departamento, construiu credibilidade do serviço junto à comunidade e isenção de articulações provindas do meio político sobreestas ações, fortalecendo a idéia de que o trabal ho realizado pelo departamento é muito importante no município, pois resulta na proteção da saúdee melhora da qualidade de vida das pessoas.

Quando comparada à produtividade do departamento em relação ao estudo de Juliano et al. ${ }^{33}$, utilizando-se um período anual para esta comparação, percebeu-se que, frente a um serviço de vigi lância sanitária que já está em GPSM , 0 departamento de Canoas apresenta expressivo número de procedimentos realizados, visto o especial momento de análise, sob o foco da implantação da GPSM neste serviço.

Referente ao citado pela gestão do departamento objeto do estudo, em alusão às principais dificuldades encontradas durante o processo de descentral ização, Lucchese ${ }^{35}$ reitera que a falta de equipe mínima é um dos grandes entraves ao avanço do processo de descentralização, tornando-se prioritária a admissão de profissionais por concurso público, para evitar a alta rotatividade e problemas de legitimidade no exercício da função da fiscalização sanitária, queexige fépública. Já segundo a interpretação de Cohen et al. ${ }^{34}$, as ações de vigilância sanitária têm sido prejudicadas em função da rotatividade dos profissionais lotados no referido órgão, sendo que grande parte destes não são do quadro efetivo, ocasionando a demissão de profissionais já capacitados por ocasião de mudanças na gestão municipal. Frente a isto, faz-se importante ressaltar os resultados encontrados no presente estudo que apontam a quase totalidade dos profissionais do departamento de vigilância sanitária de Canoas, Rio Grande do Sul, com regimedetrabalho como estatutários (concursados), bem como apresentarem el evada média de anos de tempo detraba- 
Iho no referido setor. Nesta lógica, podemos afirmar que tais resultados podem configurar um diferencial para o al cance da efetividadenas ações no serviço estudado, durante o processo de implantação da GPSM .

Perfil e características da equipe de fiscais

No que se refere à caracterização do perfil da população-alvo, se constatou a maioria feminina nos sujeitos da pesquisa e escolaridade dos profissionais com predominância de nível superior, verificando-se semelhança de resultados com 0 estudo de Araújo et al. ${ }^{36}$, no qual destacam que a grande maioria dos profissionais da equipe de vigilância sanitária que analisaram é do sexo feminino, sendo quegrande partedessa equipepossuía formação de nível superior. No entanto, tal similaridade de resultados já não é encontrada quando nos referimos à média de idade dos fiscais e renda, visto que no departamento de vigilância sanitária de Canoas, Rio Grande do Sul, os profissionais pesquisados perfazem média deidademais el evada eapresentam renda mensal superior ao detectado pelo estudo dos autores citados. Todavia, em referência à escolaridade e renda, tem-se a continuidade dos resultados de que os profissionais fiscais com maior escolaridade têm maior renda.

Acerca da satisfação da atividade profissional, no presente estudo percebeu-se que a satisfação quanto à realização das atividades profissionais é diretamenteligadaà renda. Enquanto queno estudo de Araújo et al. ${ }^{36}$ a satisfação profissional foi associada ao desgaste do trabalho, que muitas vezes resulta em confronto e até risco pessoal, sendo que os profissionais que não consideram a atividade desgastante justificavam com 0 argumento de que gostam do tipo de trabal ho que desenvolvem. Porém, a baixa remuneração salarial também é citada como motivo de insatisfação e desmotivação para o desenvolvimento do trabalho.

Verifica-se ainda que, apesar da metade dos profissionais do departamento de vigilância sanitária de Canoas compreenderem que não realizam capacitação suficiente para o pleno desempenho de suas atividades, ainda assim, em sua maioria, os mesmos profissionais entendem que é disponibilizada tal capacitação pelos órgãos competentes. De forma análoga, mas não semeIhante, Araújo et al. ${ }^{36}$ obtiveram que a maioria dos profissionais pesquisados já participaram de processos de capacitação na área de vigilância sanitária; no entanto, todos estes afirmavam terem necessidade de aprimorar seus conhecimentos na área. Este impasse pode ser discutido pela premissa de que profissionais com perfis adequados são aquel es quetêm a formação profissional compatível com as especialidades a serem desempenhadas e capacitação apropriada, sendo as equipes de trabalho em vigilância sanitária multiprofissionais, pois os campos de abrangência se inter-relacionam ${ }^{37}$.

Por outro lado, a direção do departamento de vigilância sanitária de Canoas afirma que a capacitação de seus técnicos para exercerem as atribuições provindas da implantação da GPSM já vinha sendo contemplada anteriormente ao referido processo de descentralização, de forma gradual, com a equipe de técnicos do município acompanhando os técnicos da esfera estadual durante a efetivação destas atividades.

\section{Função essencial de saúde pública no 6}

Aplicando-se a FESP no 6 na concretização dos objetivos desta pesquisa, obteve-se como resultado para os indicadores FESP 6.2 e FESP 6.3, um desempenho de medição acima da mé dia. Frente ao indicador FESP 6.4, os resultados apontam ótimo desempenho. Por sua vez, a mé dia final obtida pela medição do instrumento, considerando todos os indicadores, aponta um desempenho acima da média.

Tais resultados, quando comparados ao exercício demedição da OPAS, do mesmo instrumento nos países da América Latina, inclusiveno Brasil, que demonstram mínimo desempenho para 0 indicador FESP $6.2(0,27)$, baixo desempenho para os indicadores FESP $6.3(0,53)$ e $6.4(0,38)$, e desempenho mediano $(0,47)$ para a média final ${ }^{29}$, acabam por representar a comprovação da efetividade das ações de vigilância sanitária no departamento foco desteestudo, principalmenteno que tange a avaliação de aspectos do cumprimento das normas, mecanismos de fiscalização e assessoria técnica oferecida pela esfera estadual durante o processo de descentralização.

Confirmando esta situação, também se verifica o efetivo desempenho demedição obtido pelo departamento de Canoas, quando em análise aos resultados de medição realizada pela OPAS nos países da América Central de língua espanhola, considerando também Caribee Haiti. N esteexercício de aplicação da FESP $n$ ㅇ 6, teve-se como resultado para todos os indicadores (FESP $6.2=$ 0,36 ; FESP $6.3=0,45$; FESP $6.4=0,30$ ), bem como para a média geral da medição $(0,48)$, um desempenho considerado mediano ${ }^{29}$.

Desta forma, comparando-se os resultados 
da medição FESP obtida no presenteestudo com os exercícios de medição em países da América Latina, incluindo o Brasil, e da América Central, o desempenho apresentado de efetividade das ações de vigilância sanitária no departamento de Canoas foi acima da média.

Também pôde-se verificar outros relevantes resultados pelo intermédio da FESP $n \circ 6$, quando analisadas isoladamente as variáveis de cada indicador, consideradas mais importantes para caracterizar o objetivo específico demedição. Por exemplo, em observação ao indicador FESP 6.2, verificou-se que metade dos fiscais entrevistados compreendem que a gestão do serviço não contempla medidas para evitar manipulações degrupos de pressão externos, mas que, no entanto, a mesma gestão éágil para corrigir o abuso de poder do grupo de fiscalização, possuindo políticas e planos para prevenir estetipo decorrupção, conforme respostas de grande parcela dos entrevistados. Frente a isso, ressalta-se que a vigilância sanitária, por suas características e inserção em campo de conflito, éa prática de saúde que mais sofre as conseqüências de intervenções clientelistas ou de interesses políticos escusos ${ }^{35}$.

Seguindo esta análise das principais variáveis, observou-se quanto ao indicador FESP 6.3, que grande parte dos entrevistados entendem que 0 departamento não dispõe de recursos humanos suficientes para a fiscal ização; entretanto, a maioria deles afirma que o departamento dispõe de recursos financeiros suficientes. Não obstante, Eduardo ${ }^{38}$ afirma que historicamente as ações de vigilância sanitária não têm merecido a mesma importância que aquelas referentes à saúde individual no Brasil. E nas práticas coletivas, talvez elas sejam as mais marginalizadas pelas políticas de saúde, passando ao longo dos governos, salvo al gumas exceções, com equipes insuficientes em recursos humanos, sem acesso a treinamentos e atualizações, sem veículos para realizar as inspeções em locais mais distantes, submetidas a salários irrisórios, dentre tantos outros problemas.

Por fim, em referência ao indicador FESP 6.4, de acordo com a avaliação dos fiscais do departamento, o Estado prestou plena assessoria no processo de descentralização, apoiando com treinamentos e orientando nos procedimentos de fiscalização. Assessoria esta respaldada em regulamentação específica que atribui ao Estado a responsabilidade pela execução destas ações de suportetécnico ${ }^{39}$.

Contudo, a utilização do instrumento FESP no 6 apresentou algumas limitações, em virtude de ter sido desenvolvido pela OPAS para avaliar a prática das FESP em nível nacional, sendo que, para esta pesquisa, seu foco foi avaliar um serviço de âmbito municipal, não tendo sido encontrado outros estudos nestes moldes, o que acabou por limitar maior discussão e comparação dos resultados obtidos. Por outro lado, não se encontrou outro instrumento validado tão específico etecnicamente formulado como a FESP no 6 para se avaliar as práticas de vigilância sanitária, ao passo que se apresentou de forma adequada à utilização proposta, correspondendo satisfatoriamente ao alcance dos objetivos da pesquisa. Outrossim, a identificação específica das variáveis mais importantes dentre as diversas queconstituem cada indicador também pode ser considerada outra limitação do instrumento.

Outra possível limitação do estudo seria o inerente risco de viés de aferição dos resultados, visto a pesquisa ser baseada em coleta de informações subjetivas, somado ao fato dos sujeitos do estudo estarem avaliando o serviço no qual os próprios estão inseridos, o que poderia ocasionar uma visão não tão real dos resultados, estando o viés de pesquisa sendo produzido pela própria fonte dedados. Porém, todo o sigilo frente à identificação dos respondentes foi providenciado, não tendo sido permitido a identificação das respostas aos entrevistados, inclusive frente à direção do departamento. Considerando-se também que 0 trabalho apresenta somente a visão do profissional da vigilância sanitária, prestador do serviço, visto não ser o objetivo deste trabaIno apresentar a perspectiva do usuário, o qual sabidamente poderia disponibilizar uma dimensão mais completa a respeito da efetividade do serviço avaliado.

Entretanto, de forma gradual, a idéia da utilização das FESP para o fortalecimento da saúde pública vem sendo construída e implantada por outros pesquisadores com os mais diversos objetivos de pesquisa, todos contemplados pelo intermédio da FESP. Como exemplo, referenciamos Witt ${ }^{40}$, que pesquisou sobre o trabalho do profissional de enfermagem na atenção básica em saúde tendo como questão central as competências gerais e específicas do enfermeiro e sua contribuição para a construção do SUS, e para a constituição das FESP. Por sua vez, Santos ${ }^{41}$, utilizou a FESP no 4 para conhecer a participação dos cidadãos de um município através do conseIho municipal de saúde, na gestão do SUS. Já em âmbito nacional, podemos citar projeto do Conselho $\mathrm{N}$ acional deSecretários Estaduais deSaúde (CONASS) que promoveu medição das FESP adaptadas à gestão estadual do SUS, identifican- 
do em nível estadual as áreas de baixo desempenho na gestão do serviço, para posterior fortalecimento, superando as fragilidades apontadas com vistas à consolidação das ações de vigilância sanitária no âmbito da descentralização do SU S ${ }^{42}$.

\section{Conclusões}

Após avaliação do departamento de vigilância sanitária de Canoas, Rio Grande do Sul, durante o processo de implantação da GPSM, pode-se concluir que o serviço é efetivo baseado no instrumento de pesquisa utilizado (FESP), frente 0 desenvolvimento das ações de vigilância sanitária e que consta de estrutura e de equipe de fiscais, que respondem plenamente às demandas necessárias de suas atribuições segundo avaliação do gestor do serviço, bem como apresentam perfil semelhante às outras equipes de serviços de vigilância sanitária ${ }^{33,34,36}$.

Portanto, pode-se concluir que um serviço de vigilância sanitária que não possui regulamentos claros e efetivos, sem administração racional eágil, deficiente decanais bem delimitados de participação social, que não dispõe de corpo suficiente de funcionários qualificados e sem estrutura condizentecom a demanda queatende, certamenteserá incapaz de sinalizar claramente as regras nesta área sanitária e de garantir a sua efetiva ação por todos os agentes envolvidos, por maior que seja o montante de recursos destinados, bem como indiferente à forma de gestão relacionada à descentralização em que se encontra.

Entende-se que o processo de descentralização podeser adequadamente construído, se proceder à lógica de atribuir os devidos model os de gestão somente a projetos municipais organizados e comprovadamente capazes de gerenciar e aplicar os recursos recebidos, de forma a ressaltar a efetividade nas ações desenvolvidas através deum instrumento de avaliação. Contudo, frente a sua importância de existência e de ação, considera-se essencial a definição deum modelo ideal de gestão descentralizada e municipalizada, formulado a partir de perfis de serviços de vigilância sanitária já estruturados e com comprovados resultados de efetividade em suas ações.

Não obstante, destaca-se a necessidade de proporcionar aos trabal hadores de vigilância sanitária a possibilidade de reflexão sobre seu conhecimento a respeito de suas práticas profissionais, considerando as diferenças degrau deinstrução, de conhecimento e de capacitação, visto que são responsáveis diretos pela atual caracterização do perfil dos serviços nos quais atuam ${ }^{43}$.

\section{Colaboradores}

J S Souza trabal hou na concepção, pesquisa e redação final eAT Stein trabalhou na metodologia, orientação e revisão final. 


\section{Referências}

1. Brasil. Constituição da República Federativa do Brasil de 05 de outubro de 1988. Diário Oficial da União 1988; 5 out.

2. Costa EA. Vigilância sanitária, proteção e defesa da saúde. São Paulo: Hucitec; 1999.

3. Hirsch GJ. Fiscalização e processo administrativo sanitário à luz da Constituição da República. Rio de Janeiro: Editora Aide; 2003.

4. Costa EA. Conceitos e área de abrangência. In: Rozenfeld $\mathrm{S}$, organizador. Fundamentos de vigilância sanitária. Rio de Janeiro: Fiocruz; 2000. p. 41-47.

5. Costa EA. Vigilância sanitária, proteção e defesa da saúde. 2a ed. Brasília: Sobravime; 2004.

6. Brasil. Lei Federal no. 8.080 , de 19 de setembro de 1990. Dispõe sobre as condições para a promoção, proteção e recuperação da saúde, a organização e o funcionamento dos serviços correspondentes e dá outras providências. Diário Oficial da União 1990; 20 set.

7. Costa EA, Rozenfeld S. Constituição da vigilância sanitária no Brasil. In: Rozenfeld S, organizador. Fundamentos de vigilância sanitária. Rio de Janeiro: Fiocruz; 2000. p.15-40.

8. Souto AN. Saúde e política. A vigilância sanitária no Brasil. São Paulo: Sobravime; 2004.

9. Silva ZP, Coroa M L. Health surveillance: discursive historical. REVISA 2005; 1(1):3-15.

10. Quitério LAD. Health surveillance, productive process and health risks: the promotion of health in the concrete practice of a case of environment contamination. REVISA 2005; 1(1):79-83.

11. Augusto LGS. The challenge of the integration of the surveillances in Brazil. REVISA 2005; 1(1):75-78.

12. Novaes HMD. Evaluation of health programs, services and technologies. Rev. Saúde Pública 2000; 34(5):559-547.

13. Scatena JHG, Tanaka OY. A descentralização da saúde no estado de M ato Grosso, Brasil: financiamento e modelo de atenção. Rev Panam Salud Publica 2000; 8(4):249-242.

14. Lucchese PTR. A vigilância sanitária, segundo as normas operacionais básicas do ministério da saúde. In: Rozenfeld S, organizador. Fundamentos de vigilância sanitária. Rio de Janeiro: Fiocruz; 2000. p.99-111.

15. Brasil. Portaria no 2.203. Norma O peracional Básica do SUS - NOB SUS 01/96. Diário O ficial da União 1996; 6 nov.

16. Arretche $M, M$ arques $E$. La municipalización de la salud en Brasil: Diferencias regionales, poder de voto y estrategias del gobierno. Serie de Informes Técnicos. Washington, D.C.: Organización Panamericana de la Salud; 2001.

17. Arretche M. Financiamento federal e gestão local de políticas sociais: o difícil equilíbrio entre regulação, responsabilidades e autonomia. Cien Saude Colet 2003; 8(3):331-345.

18. Portela MC. Avaliação da qualidade em saúde. In: Rozenfeld S, organizador. Fundamentos de vigilância sanitária. Rio de Janeiro: Fiocruz; 2000. p. 259-269.

19. Donabedian A. Continuity and change in the quest for quality. Clin Perfom Qual Health Care 1996;1:916.
20. Hortale VA, Conill EM, Pedroza M. Challenges for developing a comparative analytical model for health services. Cad Saúde Pública 1999; 15(1):79-88.

21. Donabedian A. The seven pillars of quality. Arch Pathol Lab Med 1990; 114:1115-1118.

22. Pereira M G. Epidemiologia: teoria e prática. 4a ed. Rio de Janeiro: Guanabara Koogan; 2000.

23. Gray JAM. Evidence-based healthcare - How to make health policy and management decision. New York: Curchill Livingstone; 1997.

24. Fundação Instituto Brasileiro de Geografia e Estatística. Estatísticas das cidades; 2006. Disponível em: http://www.ibge.gov.br

25. Federação das Associações de Municípios do Rio Grande do Sul. Informações M unicipais; 2006. Disponível em: http://ww2.famurs.com.br

26. Organização Pan-Americana da Saúde, Organização M undial da Saúde. 126a Sessão do comitê executivo. Funções Essenciais de saúde pública.Tema 4.10 da agenda provisória. Washington, D.C.: Organização Pan-Americana da Saúde; 2000.

27. Organização Pan-Americana da Saúde, Organização Mundial da Saúde. A saúde pública nas Américas. Instrumento para a medição do desempenho das funções essenciais de saúde pública. Washington, D.C.: Organização Pan-Americana da Saúde; 2001.

28. Organização Pan-Americana da Saúde, Organização Mundial da Saúde. Medição do desempenho das funções essenciais de saúde pública. Guia para a aplicação do instrumento de desempenho das funções essenciais de saúde pública. Washington, D.C.: Organização Pan-Americana da Saúde; 2001.

29. Organização Pan-Americana da Saúde, Organização Mundial da Saúde. La salud pública en las Américas: nuevos conceptos, analises del desempeño y bases para la acción. Washington, D,C,: Organização Pan-Americana da Saúde; 2002.

30. Epidata Software Versão 3.1 [programa de computador]; 2006. Disponível em: http://www.epiinfo.it/ EpiData.htm

31. Epidata Software Analysis [programa de computador]; 2006. Disponível em: http://www.epidata.dk

32. SPSS Software [programa de computador]; 2006. Disponível em: http://www.spss.com.br

33. Juliano IA, Assis M M A. The sanitary surveillance in Feira de Santana in the health decentralization process (1998-2000). Cien Saude Colet 2004; 9(2):493-505.

34. Cohen M M, Moura M LO, Tomazelli JG. Decentralization of health surveillance actions in cities with local health management in the state of Rio de Janeiro. Rev Bras Epidemiol 2004; 7(3): 290-300.

35. Lucchese G. Globalização e regulação sanitária. Os rumos da vigilância sanitária no Brasil [tese]. Rio de Janeiro )RJ): Escola Nacional de Saúde Pública, Fundação Oswaldo Cruz; 2001.

36. Araújo LUA, Guerra MSA. Pesquisa - perfil dos profissionais de vigilância sanitária da secretaria municipal de saúde de Natal / RN. NESC/UFRN, Rede Observatório de Recursos Humanos em Saúde [periódico na internet]. [acessado 2006 Jun 17]. [cerca de 24 p.]. Disponível em: www.observatorio.nesc. ufrn.br 
37. Lefèvre $F, M$ arques $M C C$, Lefèvre $A M C$, Latorre MRDO, Balbinot R, Medeiros IY, Oliveira NGS, Seragi $L$. Social representation of health surveillance by the population of Águas de Lindóia: analysis of the perception of some considerable risks. REVISA 2005; 1(1):22-30.

38. Eduardo M BP. Vigilância sanitária. Para gestores municipais de serviços de saúde. São Paulo: Faculdade de Saúde Pública; 1998.

39. Heimann SL, organizadora. Descentralização do sistema único de saúde: trilhando a autonomia municipal. São Paulo: Sobravime; 2000.

40. Witt RR. Competências da enfermeira na atenção básica: Contribuição à construção das funções essenciais de saúde pública [tese]. Ribeirão Preto (SP): Escola de enfermagem de Ribeirão Preto, Universidade de São Paulo; 2005.
41. Santos CL. Participação popular na gestão do sistema único de saúde no município de Tramandaí-RS [dissertação]. Canoas (RS): Programa de Pós-Graduação em Saúde Coletiva, Universidade Luterana do Brasil; 2005.

42. Brasil. Conselho Nacional de Secretários de Saúde. Notícias do CONASS. Funções Essenciais de Saúde Pública; 2006. [texto na Internet]. [acessado 2007 jan 03]. [cerca de 13p.]. Disponível em: http://www. conass.com.br

43. Senna MCM, Cohen M M. Modelo assistencial e estratégia saúde da família no nível local: análise de uma experiência. Cien Saude Colet 2002; 7(3):1-14.

Artigo apresentado em 02/02/2007

Aprovado em 30/07/2007

Versão final apresentada em 18/10/2007 